\title{
Android Malware Classification by Applying Online Machine Learning
}

\author{
Abdurrahman Pektaş ${ }^{1}$, Mahmut Çavdar ${ }^{2}$, and Tankut Acarman ${ }^{2(凶)}$ \\ 1 The Scientific and Technological Research Council of Turkey, Ankara, Turkey \\ ${ }^{2}$ Computer Engineering Department, Galatasaray University, İstanbul, Turkey \\ acarmant@gmail.com
}

\begin{abstract}
A malware is deployed to execute malicious activities in the compromised operating systems. The widespread use of android smartphones with high speed Internet and permissions granted to applications for accessing internal logs provides a favorable environment for the execution of unauthorized and malicious activities. The major risk and challenge lies along classification of a large volume and variety of malware. A malware may evolve and continue to hide its malicious activies against security systems. Knowing malware features a priori and classification of a malware plays a crucial role at defending the safety and liability critical user's information. In this paper, we study android malware activities, features and apply online machine learning algorithm to classify a new android malware. We extract a fairly adequate set of malware features and we evaluate a machine learning based classification method. The runtime model is built and it can be implemented to detect variants of an android malware. The metrics illustrate the effectiveness of the proposed classification method.
\end{abstract}

\section{Introduction}

According to Internet Security Report, 1.4 billion smartphones were sold in 2015 and 83,3\% phones were running Android, [1]. Their users may save information about their personal identities, online payment system access and user's credentials. Malware authors, cyber criminals aim to steal these information via the distribution and installation of android applications. Overall, 3.3 million applications were classified as malware in 2015. Malware authors deliver this large variety and volume of malicious software by using advanced obfuscation techniques. Therefore, behavior-based malware analysis and classification of a malware sample to its original family plays a crucial and timely role at taking security and protection counter measures.

Android is a complete operating system that uses Android application (app) package (APK) for distribution and installation of mobile apps. APK file contains components which share a set of resources like database, preference, files, classes compiled in the dex file format, etc., App components are divided in four categories: activities handling the user interaction; services carrying out background tasks; content providers managing app's data; broadcast receivers

(C) The Author(s) 2016

T. Czachórski et al. (Eds.): ISCIS 2016, CCIS 659, pp. 72-80, 2016.

DOI: $10.1007 / 978-3-319-47217-1 \_8$ 
Table 1. List of system commands and command's execution frequency by our malware test set

\begin{tabular}{l|l|l}
\hline Command & Description & Frequency \\
\hline /system/bin/cat (i.e. cat) & display files & 33 \\
\hline logCat & $\begin{array}{l}\text { reads the compressed logging files and } \\
\text { outputs human-readable messages } \\
\text { verifies IP-level connectivity by using } \\
\text { ICMP }\end{array}$ & 13 \\
\hline chmod & $\begin{array}{l}\text { used to change the permissions of files } \\
\text { or directories }\end{array}$ & 4 \\
\hline ln & creates a link to an existing file & 3 \\
\hline mount & attaches additional filesystem & 2 \\
\hline echo & outputs text to the screen or a file & 2 \\
\hline su & $\begin{array}{l}\text { used to execute commands with the } \\
\text { privileges of another account }\end{array}$ & 2 \\
\hline id & $\begin{array}{c}\text { print user ID and group ID of the } \\
\text { current user }\end{array}$ & 2 \\
\hline
\end{tabular}

assuring communications between components, app's, even more Android OS. The manifest declares the app's components and how they interact. Also user permissions required by the apps are placed in the manifest file. Android is a privilege-separated operating system, in which each application runs with a distinct system identity (Linux user ID and group ID). Parts of the system are also separated into distinct identities. Linux thereby isolates applications from each other and from the system.

Several commands can be used to infect Android devices. For example, Cat command, i.e., System/bin/cat displays files in the system and it can be executed for malicious purposes. The command-line tool LogCat can be used for viewing the internal logs. Log messages may include privacy-related information. An app can access the log file by giving every app the READ_LOGS permission with aid of the chmod command. The list of commands is described in Table 1.

In line with the emerging market of android smartphones, detection and classification of its malware has attracted a lot of attention. Static analysis of the executables by using commands, and modelling of malware features by using permissions and API calls is presented for the detection of a malware in [2,3]. K-means algorithm for clustering and a decision tree learning algorithm for classification of a malware is presented by monitoring various permission based features and events extracted from applications in [4]. A learning model database is obtained by collecting the extracted features and N-gram signatures are created in [5]. Text mining and information retrieval is applied for the static analysis of a malware in [6]. In [7], a heuristics approach by using 39 different behaviour flags such as Java API calls, presence of embedded executables and code size is developed to determine whether an application is malicious or not. A deep learning for automatic generation of malware signature is studied to detect a majority of new variants of a malware in [8]. And, a detection model is trained with the information gathered via the communication among components. A security framework has been 
deployed by an European project called NEMESYS for gathering and analyzing information about the nature of cyber-attacks targeting mobile devices and presented a model-based approach for detection of anomalies [9-11].

The paper is organized as follows: In Sect. 2, we present the selected features. In Sect. 3, we implement online machine learning algorithm to the classification of malware samples and we evaluate the results. Finally, we conclude our paper.

\section{Feature Set}

Cuckoo Sandbox is an open source analysis system and relies on virtualization technology to run a given file, [12]. It can analyze both executable and nonexecutable files and monitor the run-time activities. In this study, we extracted

Table 2. Features and their types

\begin{tabular}{l|l|l}
\hline Feature category & Type & Value \\
\hline commands & String & /system/bin/cat \\
\hline services & String & com.houseads.AdService, \\
\cline { 3 - 3 } & & com.applovin.sdk.AppLovinService,' \\
\hline pingerprint & String & getSimCountryIso, getDeviceId, getLine1Number \\
\hline data_leak & String & INTERNET, ACCESS_NETWORK_STATE, \\
& & READ_PHONE_STATE, GET_ACCOUNTS \\
\hline file_accessed & String & getAccounts \\
\hline httpConnections & String & /proc/net/if_inet6, /proc/meminfo ... \\
\hline send_sms & String & http://houseads.eu/ads/new_user.php?id=147 \\
\hline receive_sms & & \&im=351451208401216 \&l=en\&c=us\&bm \\
\hline read_sms & & =Nexus+5\&bv=4.1.2\&v=4.2\&ct=UMTS \\
\hline call_phone & Boolean & FALSE \\
\hline ap_execute_shell_commands & Boolean & TRUE \\
\hline app_queried_account_info & Boolean & TRUE \\
\hline app_queried_installed_apps & Boolean & FALSE \\
\hline app_queried_phone_number & Boolean & TRUE \\
\hline app_queried_private_info & Boolean & FALSE \\
\hline app_recording_audio & Boolean & FALSE \\
\hline app_registered_receiver_runtime & Boolean & TRUE \\
\hline app_uses_location & Boolean & FALSE \\
\hline embedded_apk & Boolean & FALSE \\
\hline is_reflectionamative_code & Boolean & TRUE \\
\hline & Boolean & FALSE \\
\hline & Boolean & TRUE \\
\hline & & FALSE \\
\hline
\end{tabular}


Table 3. Top 20 requested permissions

\begin{tabular}{l|l}
\hline Permissions & Frequency \\
\hline INTERNET & 867 \\
\hline READ_PHONE_STATE & 826 \\
\hline WRITE_EXTERNAL_STORAGE & 764 \\
\hline ACCESS_NETWORK_STATE & 744 \\
\hline SEND_SMS & 565 \\
\hline INSTALL_SHORTCUT & 535 \\
\hline ACCESS_WIFI_STATE & 524 \\
\hline WAKE_LOCK & 473 \\
\hline RECEIVE_BOOT_COMPLETED & 420 \\
\hline VIBRATE & 382 \\
\hline RECEIVE_SMS & 348 \\
\hline GET_TASKS & 337 \\
\hline WRITE_SETTINGS & 306 \\
\hline READ_SMS & 285 \\
\hline ACCESS_COARSE_LOCATION & 281 \\
\hline READ_SETTINGS & 278 \\
\hline CHANGE_WIFI_STATE & 277 \\
\hline ACCESS_FINE_LOCATION & 270 \\
\hline CALL_PHONE & 215 \\
\hline SYSTEM_ALERT_WINDOW & 182 \\
\hline
\end{tabular}

the most significant and distinguishing behavioral features from the Cuckoo's analysis report. The list of android malware features is given in Table 2 . The permissions requested by the applications are ranked according to their persistency in Table 3 .

\section{Implementation}

The testing malware dataset is obtained from "VirusShare Malware Sharing Platform" ([13]), which provides a huge amount of different type malware including PE, HTML, Flash, Java, PDF, APK etc. All experiments were conducted under the Ubuntu 14.04 Desktop operating system with $\operatorname{Intel}(\mathrm{R}) \operatorname{Core}(\mathrm{TM})$ i5-2410M@2.30 GHz processor and 2 GB of RAM. The analysis with 5 guest machines took 5 days to analyze approximately 2000 samples. For labeling malware samples, we used Virustotal, an online web-based multi anti-virus scanner, [14]. The malware classes along their class-specific measures are given in Table 4. 
Table 4. Malware families and their class-specific measures

\begin{tabular}{l|r|l|l|l|l|l}
\hline Family & Code & $\#$ & Recall & Specificity & Precision & $\begin{array}{l}\text { Balanced } \\
\text { accuracy }\end{array}$ \\
\hline android.trojan.fakeinst & 1 & 193 & 0.94 & 0.98 & 0.94 & 0.96 \\
\hline android.riskware.smsreg & 2 & 104 & 0.67 & 0.99 & 0.86 & 0.83 \\
\hline android.trojan.agent & 3 & 79 & 0.60 & 1.00 & 1.00 & 0.80 \\
\hline android.adware.gingermaster & 4 & 74 & 0.67 & 0.99 & 0.80 & 0.83 \\
\hline android.adware.adwo & 5 & 69 & 0.83 & 1.00 & 1.00 & 0.92 \\
\hline android.trojan.smssend & 6 & 66 & 1.00 & 0.84 & 0.35 & 0.92 \\
\hline android.trojan.smskey & 7 & 48 & 0.25 & 1.00 & 1.00 & 0.63 \\
\hline android.adware.utchi & 8 & 45 & 1.00 & 1.00 & 1.00 & 1.00 \\
\hline android.trojan.clicker & 9 & 37 & 1.00 & 0.99 & 0.75 & 0.99 \\
\hline android.adware.appquanta & 10 & 34 & 1.00 & 1.00 & 1.00 & 1.00 \\
\hline android.adware.plankton & 11 & 34 & 0.50 & 1.00 & 1.00 & 0.75 \\
\hline android.trojan.fakeapp & 12 & 19 & 1.00 & 1.00 & 1.00 & 1.00 \\
\hline android.trojan.boqx & 13 & 18 & 0.50 & 1.00 & 1.00 & 0.75 \\
\hline android.trojan.killav & 14 & 17 & 1.00 & 1.00 & 1.00 & 1.00 \\
\hline android.riskware.tocrenu & 15 & 14 & 0.50 & 1.00 & 1.00 & 0.75 \\
\hline android.exploit.gingerbreak & 16 & 12 & 1.00 & 1.00 & 1.00 & 1.00 \\
\hline android.trojan.bankun & 17 & 12 & 1.00 & 1.00 & 1.00 & 1.00 \\
\hline android.trojan.smsspy & 18 & 11 & 1.00 & 1.00 & 1.00 & 1.00 \\
\hline
\end{tabular}

\subsection{Online Classification Algorithms}

In general, an online learning algorithm works in a sequence of consecutive rounds. At round $t$, the algorithm takes an instance $\boldsymbol{x}_{t} \in \mathbb{R}^{d}$, d-dimensional vector, as input to make the prediction $\hat{y}_{t} \in\{+1,-1\}$ (for binary classification) regarding to its current prediction model. After predicting, it receives the true label $y_{t} \in\{+1,-1\}$ and updates its model (a.k.a. hypothesis) based on prediction loss $\ell\left(y_{t}, \hat{y}_{t}\right)$ meaning the incompatibility between prediction and actual class. The goal of online learning is to minimize the total number of incorrect predictions; $\operatorname{sum}\left(t: y_{t} \neq \hat{y}_{t}\right)$. Pseudo-code for generic online learning is given in Algorithm-1.

\subsection{Classification Metrics}

To evaluate the proposed method, the following class-specific metrics are used: precision, recall (a.k.a. sensitivity), specificity, balanced accuracy, and overall accuracy (the overall correctness of the model). Recall is the probability for a sample in class $c$ to be classified correctly. On the contrary, specificity is 


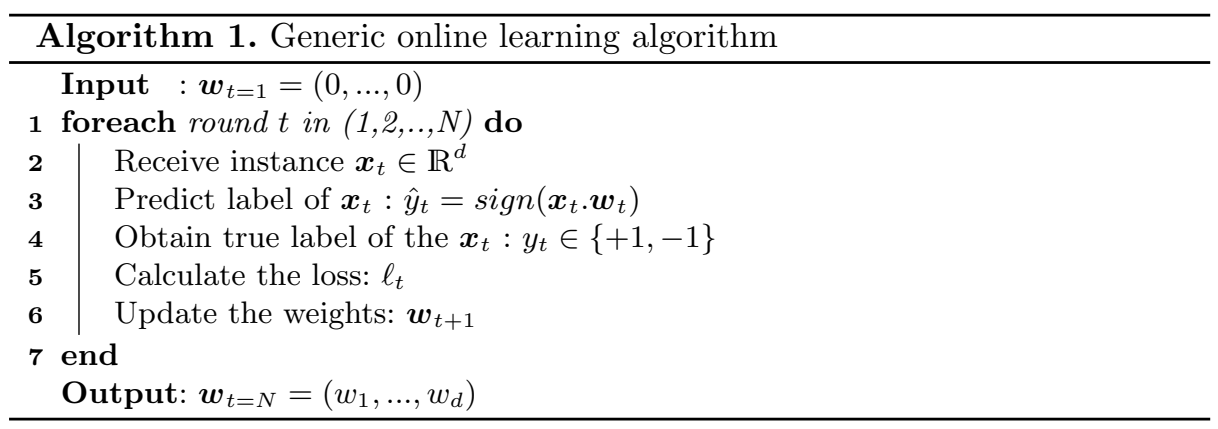

the probability for a sample not in class $c$ to be classified correctly. The metrics are given as follows:

$$
\begin{aligned}
\text { precision } & =\frac{t p}{t p+f p} \\
\text { recall } & =\frac{t p}{t p+f n} \\
\text { specificity } & =\frac{t n}{t n+f p} \\
\text { balanced accuracy } & =\frac{\text { recall }+ \text { specificity }}{2}=\frac{1}{2}\left(\frac{t p}{t p+f n}+\frac{t n}{t n+f p}\right) \\
\text { accuracy } & =\frac{\text { correctly classified instances }}{\text { total number of instances }}
\end{aligned}
$$

For instance, consider a given class $c$. True positives $(t p)$ refer to the number of the samples in class $c$ that are correctly classified while true negatives $(t n)$ are the number of the samples not in class $c$ that are correctly classified. False positives $(f p)$ refer the number of the samples not in class $c$ that are incorrectly classified. Similarly, false negatives $(f n)$ are the number of the samples in class $c$ that are incorrectly classified. The terms positive and negative indicate the classifier's success, and true and false denotes whether or not the prediction matches with ground truth label.

\subsection{Testing Accuracy Results}

The accuracy of testing is computed subject to different value of regularization weight parameter. The regularization weight parameter is denoted by $C$ and determines the size of weight change at each iteration. A larger value means a possibility of a higher change in the updated weight vector and the model is created faster. But as a consequence, the model becomes more dependent to the training set and more susceptible to noise data. 10-fold cross-validation approach is used. The class-wise results for the most successful algorithm (i.e. Confidenceweighted linear classification in [15]) according to the different weight $C$ are given in Table 5 . 
Table 5. Classification accuracy versus different regularization weight parameter

\begin{tabular}{l|l|l|l|l|l|l}
\hline$C=1$ & $C=2$ & $C=3$ & $C=4$ & $C=5$ & $C=10$ & $C=100$ \\
\hline 0.81 & 0.83 & 0.84 & 0.89 & 0.80 & 0.78 & 0.76 \\
\hline
\end{tabular}

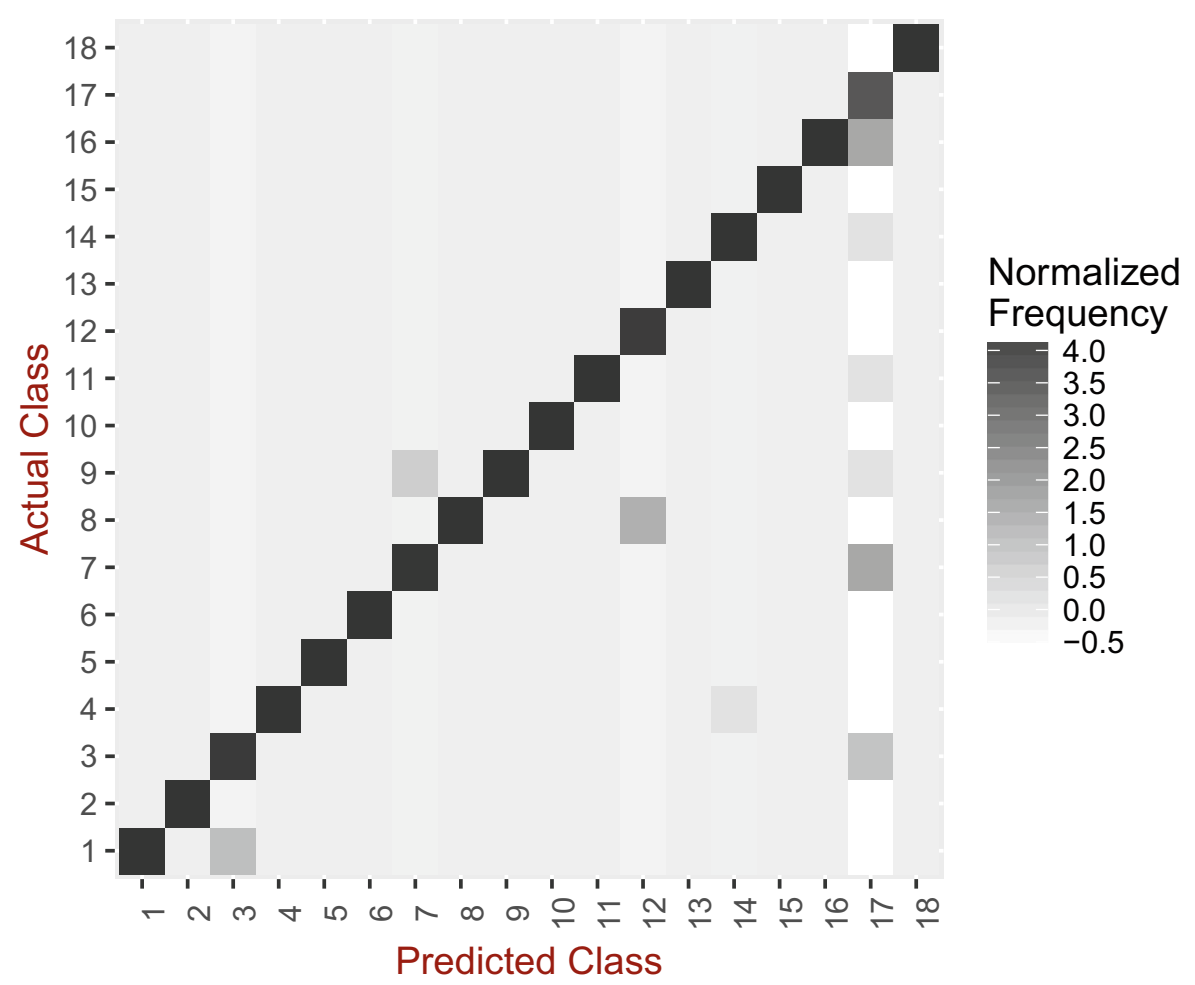

Fig. 1. Normalized confusion matrix

To analyze how well the classifier can recognize instance of different classes, we created the confusion matrix as shown in Fig. 1. The confusion matrix displays the number of correct and incorrect predictions made by the classifier with respect to ground truth (actual classes). The diagonal elements in the matrix represent the number of correctly classified instances for each class, while the off-diagonal elements represent the number of misclassified elements by the classifier. The higher the diagonal values of the confusion matrix are, the better the model fits the dataset (higher accuracy in individual family prediction). Since android.trojan.bankun family combines many functionalities executed also by other families in our dataset, android.trojan.agent, android.trojan.smskey and android.exploit.gingerbreak are incorrectly estimated as android.trojan.bankun. 


\section{Conclusions}

This paper addresses the challenge of classifying android malware samples by using runtime artifacts while being robust to obfuscation. The presented classification system is usable on a large scale in real world due to its online machine learning methodology. The proposed method uses run-time behaviors of an executable to build the feature vector. We evaluated an online machine learning algorithm with 2000 samples belonging to 18 families. The results of this study indicate that runtime behavior modeling is a useful approach for classifying an android malware.

Acknowledgments. The authors gratefully acknowledge the support of Galatasaray University, scientific research support program under grant \#16.401.004.

Open Access. This chapter is distributed under the terms of the Creative Commons Attribution 4.0 International License (http://creativecommons.org/licenses/by/ $4.0 /$ ), which permits use, duplication, adaptation, distribution and reproduction in any medium or format, as long as you give appropriate credit to the original author(s) and the source, a link is provided to the Creative Commons license and any changes made are indicated.

The images or other third party material in this chapter are included in the work's Creative Commons license, unless indicated otherwise in the credit line; if such material is not included in the work's Creative Commons license and the respective action is not permitted by statutory regulation, users will need to obtain permission from the license holder to duplicate, adapt or reproduce the material.

\section{References}

1. Internet Security Threat Report (2016) Available via Symantec. https://www. symantec.com/content/dam/symantec/docs/reports/istr-21-2016-en.pdf. Cited 15 Jun 2016

2. Schmidt, A.D., Bye, R., Schmidt, H.G., Clausen, J., Kiraz, O.: Static analysis of executables for collaborative malware detection on Android. In: 2009 IEEE International Conference on Communications, Dresden, pp. 1-5 (2009)

3. Peiravian, N., Zhu, X.: Machine learning for android malware detection using permission and API calls. In: Proceedings of the ICTAI 2013, The IEEE 25th International Conference on Tools with Artificial Intelligence, pp. 300-305 (2013)

4. Aung, Z., Zaw, W.: Permission-based android malware detection. Int. J. Scient. Technol. Res. 2, 228-234 (2013)

5. Dhaya, R., Poongodi, M.: Detecting software vulnerabilities in android using static analysis. In: Proceedings of ICACCCT, Communication IEEE International Conference on Advanced Communication Control and Computing Technologies, pp. 915-918 (2014)

6. Tangil, G.S., Tapiador, J.E., Lopez, P.P., Blasco, J.: A text mining approach to analyzing and classifying code structures in android malware families. Expert Syst. Appl. 4, 1104-1117 (2014)

7. Apvrille, A., Strazzere, T.: Reducing the window of opportunity for Android malware gotta catch em all. J. Comput. Virol. 8, 61-71 (2012) 
8. Xu, K., Li, Y., Deng, R.H.: ICCDetector: ICC-based malware detection on Android. Inf. Forensics Sec. 11, 1252-1264 (2016)

9. Abdelrahman, O.H., Gelenbe, E., Görbil, G., Oklander, B.: Mobile network anomaly detection and mitigation: the NEMESYS approach. In: Gelenbe, E., Lent, R. (eds.) Information Sciences and Systems. LNEE, vol. 264, pp. 429-438. Springer, Switzerland (2013). doi:10.1007/978-3-319-01604-7_42

10. Gelenbe, E., Görbil, G., Tzovaras, D., Liebergeld, S., Garcia, D., Baltatu, M., Lyberopoulos, G.: NEMESYS: enhanced network security for seamless service provisioning in the smart mobile ecosystem. In: Information Sciences and Systems (2013). doi:10.1007/978-3-319-01604-7_36

11. Gelenbe, E., Görbil, G., Tzovaras, D., Liebergeld, S., Garcia, D., Baltatu, M., Lyberopoulos, G.: Security for smart mobile networks: the NEMESYS approach. In: Proceedings of the Global High Tech Congress on Electronics, pp. 63-69. IEEE (2013)

12. Cuckoo Sandbox (2016). cuckoosandbox.org. Cited 15 Jun 2016

13. Virusshare: Malware Sharing Platform (2016). http://www.virusshare.com/

14. Virustotal: An online multiple AV Scan Service (2016). http://www.virustotal. $\mathrm{com} /$

15. Dredze, M., Crammer, K., Pereira, F.: Confidence-weighted linear classification. In: Proceedings of the 25th International Conference on Machine Learning, pp. 264-271. ACM (2008) 